\title{
FUNCTIONAL EQUATIONS FOR HIGHER LOGARITHMS
}

\author{
HERBERT GANGL
}

\begin{abstract}
Following earlier work by Abel and others, Kummer gave functional equations for the polylogarithm function $\operatorname{Li}_{m}(z)$ up to $m=5$ in 1850, but no example for larger $m$ was known until recently. We give the first genuine 2-variable functional equation for the 7-logarithm. We investigate and relate identities for the 3-logarithm given by Goncharov and Wojtkowiak and deduce a certain family of functional equations for the 4-logarithm.
\end{abstract}

\section{INTRODUCTION}

1.1. An essential property of the logarithm is its functional equation

$$
\log (x y)=\log (x)+\log (y) .
$$

An essential property of the dilogarithm, which is defined by the power series

$$
\operatorname{Li}_{2}(z)=\sum_{n=1}^{\infty} \frac{z^{n}}{n^{2}} \quad, \quad|z|<1,
$$

and can be analytically continued to $\mathbb{C}-[1, \infty)$, is the 5 -term relation (note the involutary symmetry $\left.(x, y) \mapsto\left(\frac{1-x}{1-y^{-1}}, \frac{1-y}{1-x^{-1}}\right)\right)$

$$
\mathrm{Li}_{2}(x y)-\mathrm{Li}_{2}(x)-\mathrm{Li}_{2}(y)-\mathrm{Li}_{2}\left(\frac{1-x}{1-y^{-1}}\right)-\mathrm{Li}_{2}\left(\frac{1-y}{1-x^{-1}}\right)=(\text { elementary }),
$$

which was found - in a lot of different forms - by Spence, Abel and many others (cf. [6], Chapter I). Here the "elementary" right hand side consists a sum of products of logarithms.

In 1840, Kummer [5] found functional equations for higher (poly-)logarithms

$$
\operatorname{Li}_{m}(z)=\sum_{n=1}^{\infty} \frac{z^{n}}{n^{m}}, \quad|z|<1,
$$

(which can be likewise analytically continued) up to $m \leq 5$. His method does not extend to higher $m$, though - for a more detailed statement we refer the reader to Wechsung's paper [10], where more functional equations in Kummer's spirit are derived and where it is shown that there are no such "Kummer-type" equations for $m>5$.

Other efforts in the direction of finding new equations, even with the help of a computer, seemed also to be restricted to $m \leq 5$ (cf., e.g., [8], [9], where a number of new 1-variable equations in that range were given).

In our thesis [2] we gave the first (non-trivial) functional equations for $m=6$ (in fact, a whole family of equations in two variables) and for $m=7$, as well as many new examples for $m \leq 5$ (some of those results had already been included in [14] and in Chapter 16 of [7]). The main tool in our-computer-aided-investigation was Zagier's criterion for functional equations of the associated one-valued versions of the $m$-logarithm (cf. [14], Proposition 1 and Proposition 3).

The relation of polylogarithms and algebraic $K$-theory, and in particular Zagier's conjecture on special values of Dedekind zeta functions, have made it clear 
that the question of finding functional equations for higher polylogarithms - as well as to relate them to each other - is a central one (cf., e.g., [15]). For $m=3$, Goncharov [4] found a basic functional equation as the key step in his proof of Zagier's conjecture for this case, while Wojtkowiak [12] gave a whole family of relations. The relationship between these equations has not been clarified so far.

The contents of the paper are as follows: in $\S 2$, we recall a general functional equation for the dilogarithm, which is found to have some "companion" for the trilogarithm (Proposition 3.1) which in turn specializes to Wojtkowiak's trilogarithm equations (Corollary 3.2). We restate (in §3.1) Goncharov's equation in increasingly symmetric ways and relate it (in $\S 3.2$ and $\S 3.3$ ) to Wojtkowiak's equations - the combining link being a 34-term equation, in fact a very special case of Wojtkowiak's equation which turns out to be equivalent to Goncharov's one. In $\S 4$, we state a family of 4-logarithm equations, a uniform and rather simple proof of which is given at the end of the paper $(\S 6)$. Finally, in $\S 5$, we present our "highscore result" (obtained in 1992): a functional equation for the 7-logarithm in two variables (with 274 terms).

\section{A general Dilogarithm EQUATion}

2.1. Recall that the function $\operatorname{Li}_{m}(z)$ is a many-valued function on $\mathbb{C}-\{0,1\}$ but that one has the one-valued continuous function $\mathcal{L}_{m}: \mathbb{C} \rightarrow \mathbb{R}$ given by

$$
\mathcal{L}_{m}(z)=\Re_{m}\left(\sum_{r=0}^{m} \frac{2^{r} B_{r}}{r !} \log ^{r}|z| \operatorname{Li}_{m-r}(z)\right), \quad|z| \leq 1, z \notin\{0,1\},
$$

where $\Re_{m}$ denotes the real part for $m$ odd and the imaginary part for $m$ even, and $B_{r}$ the $r$-th Bernoulli number $\left(B_{0}=1, B_{1}=-1 / 2, B_{2}=1 / 6, \ldots\right)$. For $|z|>1, \mathcal{L}_{m}(z)$ is given by the functional equation $\mathcal{L}_{m}(z)=(-1)^{m-1} \mathcal{L}_{m}(1 / z)$, while for $z \in\{0,1, \infty\}$ one extends the function by continuity. For $m=2$, this is the famous Bloch-Wigner dilogarithm (cf., e.g., [1]). For $m>2$, one-valued versions of $\mathrm{Li}_{m}$ were introduced by Ramakrishnan, Wojtkowiak and Zagier. The above function $\mathcal{L}_{m}(z)$ was introduced by Zagier [14] who denoted it $P_{m}(z)$. It has the advantage that the corresponding functional equations of $\mathrm{Li}_{m}$ become "clean" for $\mathcal{L}_{m}$, e.g., in the 5 -term relation stated above the elementary term on the right hand side disappears when we replace $\mathrm{Li}_{2}$ by $\mathcal{L}_{2}$.

Note that we can consider $\mathcal{L}_{m}$ as a function on $\mathbb{C}[\mathbb{C}]$, the formal $\mathbb{C}$-linear combinations of elements in $\mathbb{C}$, by extending it linearly.

2.2. There is a general functional equation for the dilogarithm found by Rogers - for a polynomial $\phi$ without constant term - and generalized by Wojtkowiak [12] and Zagier [16] to any rational function $\phi$ (cf. also Wechsung [10], [11]). For $m \in \mathbb{N}$ put

$$
\widetilde{\mathcal{L}_{m}}(x, y, z, w)=\mathcal{L}_{m}(\operatorname{cr}(x, y, z, w))=\mathcal{L}_{m}\left(\frac{x-z}{x-w} \frac{y-w}{y-z}\right),
$$

where cr denotes the cross ratio.

Theorem 2.1. Let $\phi: \mathbb{P}_{\mathbb{C}}^{1} \rightarrow \mathbb{P}_{\mathbb{C}}^{1}$ be a rational function, $\alpha, B, C, D \in \mathbb{P}^{1}(\mathbb{C})$. Then, denoting $\operatorname{deg}(\phi)$ the degree of $\phi$ and putting $A=\phi(\alpha)$, we obtain

$$
\sum_{\beta, \gamma, \delta} \widetilde{\mathcal{L}_{2}}(\alpha, \beta, \gamma, \delta)=\operatorname{deg}(\phi) \cdot \widetilde{\mathcal{L}_{2}}(A, B, C, D),
$$

where $\beta, \gamma$ and $\delta$ run through the preimages of $B, C$ and $D$, respectively, with multiplicities. 
The proposition contains as a special case the 5-term relation (e.g., if we take $\phi(z)=z(1-z)$ and $B=1, C=0, D=\infty)$ and in fact many other known equations. A functional equation which is not covered by the proposition (and presumably the only one, essentially) is the one relating $z$ and $\bar{z}$, its complex conjugate, via $\mathcal{L}_{2}(\bar{z})=-\mathcal{L}_{2}(z)$. We expect that the latter, together with the equation in the above proposition, generates all functional equations for the dilogarithm. In fact, a result by Wojtkowiak [13] states that all functional equations for the dilogarithm with arguments $\mathbb{C}$-rational expressions in finitely many variables can be written as a sum of 5 -term relations.

\section{TRILOGARITHM EQUATIONS}

The following equation for the trilogarithm was found by symmetrizing a functional equation given by Wojtkowiak [12].

Theorem 3.1. Let $\phi: \mathbb{P}_{\mathbb{C}}^{1} \rightarrow \mathbb{P}_{\mathbb{C}}^{1}$ be a rational function, $A_{i}, B_{j}, C_{k}, D_{l} \in \mathbb{P}^{1}(\mathbb{C})$, $i, j, k, l \in\{1,2\}$. Then, denoting $\operatorname{deg}(\phi)$ the degree of $\phi$, we have

$$
\sum_{i, j, k, l}(-1)^{i+j+k+l}\left(\sum_{\alpha_{i}, \beta_{j}, \gamma_{k}, \delta_{l}} \widetilde{\mathcal{L}_{3}}(\alpha, \beta, \gamma, \delta)-\operatorname{deg}(\phi) \cdot \widetilde{\mathcal{L}_{3}}\left(A_{i}, B_{j}, C_{k}, D_{l}\right)\right)=0,
$$

where $\alpha_{i}, \beta_{j}, \gamma_{k}$ and $\delta_{l}$ run through the preimages of $A_{i}, B_{j}, C_{k}$ and $D_{l}$, respectively, with multiplicities.

Wojtkowiak's original equation [12], pp.226-227, (a related equation had been found earlier by Wechsung, cf. [11], §.4) is obtained by specializing the equation from the proposition above and can be stated in a simpler form as follows:

Corollary 3.2. Let $A, B, C \in \mathbb{P}^{1}(\mathbb{C}), \phi: \mathbb{P}_{\mathbb{C}}^{1} \rightarrow \mathbb{P}_{\mathbb{C}}^{1}$ a rational function of degree $n$ and $x$ an independent variable.

$$
\begin{aligned}
& \text { Let } \phi^{-1}(A)=\left\{\alpha_{i}\right\}_{i=1}^{n}, \phi^{-1}(B)=\left\{\beta_{i}\right\}_{i=1}^{n} \text { and } \phi^{-1}(C)=\left\{\gamma_{i}\right\}_{i=1}^{n} \text {. Then } \\
& \widetilde{\mathcal{L}_{3}}(\phi(x), C, B, A)-\sum_{i, j, k=1}^{n} \widetilde{\mathcal{L}_{3}}\left(x, \gamma_{i}, \beta_{j}, \alpha_{k}\right) \\
& -\sum_{i, j, k=1}^{n} \widetilde{\mathcal{L}_{3}}\left(x, \alpha_{i}, \alpha_{j}, \gamma_{k}\right)-\sum_{i, j, k=1}^{n} \widetilde{\mathcal{L}_{3}}\left(x, \beta_{i}, \beta_{j}, \gamma_{k}\right) \\
& +\sum_{i, j, k=1}^{n} \widetilde{\mathcal{L}_{3}}\left(x, \alpha_{i}, \alpha_{j}, \beta_{k}\right)+\sum_{i, j, k=1}^{n} \widetilde{\mathcal{L}_{3}}\left(x, \beta_{i}, \beta_{j}, \alpha_{k}\right) \\
& =\text { const, }
\end{aligned}
$$

i.e., the expression on the left hand side is independent of $x$.

Here it is understood that $\alpha_{i}, \beta_{j}$ and $\gamma_{k}$ run through all preimages (counted with multiplicity) of $A, B$ and $C$, respectively.

\subsection{Around Goncharov's equation.}

3.1.1. The original description. Goncharov [4] found a beautiful interpretation of certain functional equations in terms of configuration spaces, and as a crucial byproduct he provided an equation for the trilogarithm in three variables $\alpha_{i}$ for which he gave a threefold symmetry. We reproduce it here using the shorthand 
$\beta_{i}=1-\alpha_{i}+\alpha_{i} \alpha_{i-1}(i=1,2,3)$, where indices are understood modulo 3: form the formal linear combination $\gamma\left(\alpha_{1}, \alpha_{2}, \alpha_{3}\right) \in \mathbb{Z}\left[\mathbb{Q}\left(\alpha_{1}, \alpha_{2}, \alpha_{3}\right)\right]$ given by

$$
\begin{aligned}
\gamma\left(\alpha_{1}, \alpha_{2}, \alpha_{3}\right)= & \sum_{i=1}^{3}\left(\left[\frac{1}{\alpha_{i}}\right]+\left[\beta_{i}\right]+\left[\frac{\alpha_{i} \alpha_{i-1}}{\beta_{i}}\right]+\left[\frac{\beta_{i}}{\beta_{i+1} \alpha_{i+2}}\right]+\left[-\frac{\beta_{i} \alpha_{i+1}}{\beta_{i+1}}\right]\right) \\
& +\left[-\frac{1}{\alpha_{1} \alpha_{2} \alpha_{3}}\right]-\sum_{i=1}^{3}\left(\left[\frac{\beta_{i}}{\alpha_{i-1}}\right]+\left[\frac{\beta_{i}}{\beta_{i+1} \alpha_{i} \alpha_{i-1}}\right]+[1]\right) .
\end{aligned}
$$

Proposition 3.3. With the above notation, we have

$$
\mathcal{L}_{3}\left(\gamma\left(\alpha_{1}, \alpha_{2}, \alpha_{3}\right)\right)=0 .
$$

Since there are 22 non-constant terms occurring in $\gamma\left(\alpha_{1}, \alpha_{2}, \alpha_{3}\right)$, we will refer to it as 22-term (or Goncharov's) relation.

3.1.2. A more symmetric description. Since Goncharov's equation plays such a central role for the theory, it seems worthwhile to analyze its structure a bit further. There is actually a much bigger symmetry group $G$ (of order 192) than the cyclic group on three letters acting on the set of arguments and dividing the 22 nonconstant terms into 2 orbits, one of length 16 (corresponding to the 15 terms in the first sum in (3.1) plus the single term $\left.\left[-\frac{1}{\alpha_{1} \alpha_{2} \alpha_{3}}\right]\right)$, the other one of length 6 (corresponding to the second sum, with the exclusion of the constant terms [1]).

$G$ is generated by two involutions

$$
\pi_{1}:\left(\alpha_{1}, \alpha_{2}, \alpha_{3}\right) \mapsto\left(\alpha_{1}, \alpha_{2},-\frac{\beta_{1}}{\alpha_{1} \beta_{3}}\right), \quad \pi_{2}:\left(\alpha_{1}, \alpha_{2}, \alpha_{3}\right) \mapsto\left(\frac{1}{\alpha_{1}}, \frac{1}{\alpha_{3}}, \frac{1}{\alpha_{2}}\right),
$$

together with the obvious symmetry of order 3 (shifting the indices mod 3 ). The set consisting of the arguments in the sixteen terms of the first orbit is transformed either into itself (e.g., via $\pi_{1}$ ) or into the set of inverses (e.g., via $\pi_{2}$ ). We give a presentation with more obvious symmetries: let $t_{1}, \ldots, t_{4}$ be four variables, subject to the constraint $\prod_{i} t_{i}=1$. Then the following element in $\mathbb{Q}\left(t_{1}, t_{2}, t_{3}\right)$ is annihilated by $\mathcal{L}_{3}$ for each (meaningful) evaluation of $t_{1}, t_{2}, t_{3}$ in $\mathbb{C}\left(\right.$ with $\left.t_{4}=\left(t_{1} t_{2} t_{3}\right)^{-1}\right)$ :

$\sum_{i}\left[t_{i}\right]+\sum_{\substack{i, j \\ i \neq j}}\left[\frac{1-t_{i}}{1-t_{j}^{-1}}\right]-\frac{1}{4} \sum_{\substack{i, j \\ i \neq j}}\left[t_{i} t_{j}\right]-\frac{1}{8} \sum_{\{i, j, k, l\}=\{1,2,3,4\}}\left[\frac{\left(1-t_{i}\right)\left(1-t_{j}\right)}{\left(1-t_{k}^{-1}\right)\left(1-t_{l}^{-1}\right)}\right]-3[1]$.

The obvious $\mathcal{S}_{4}$-action on the set of arguments (where we identify $[z]$ and $[1 / z]$ ), together with the involution (cf. $\S 1.1$ )

$$
t_{i} \mapsto \frac{1-t_{i}}{1-t_{i+2}^{-1}} \quad(i \bmod 4)
$$

generates the symmetry group $G$ (of order 192) mentioned above.

3.1.3. A yet more symmetric description. Consider the finite group $G^{\prime}$ (of order 96) of automorphisms of $\mathbb{Q}\left(y_{1}, y_{2}, y_{3}, z_{1}, z_{2}, z_{3}\right)$, generated by $G^{\prime}=\langle g, h\rangle$, where

$$
\begin{aligned}
& g:\left(y_{1}, y_{2}, y_{3}, z_{1}, z_{2}, z_{3}\right) \mapsto\left(\frac{1}{y_{1}}, z_{2}, z_{3}, z_{1}, y_{2}, y_{3}\right), \\
& h:\left(y_{1}, y_{2}, y_{3}, z_{1}, z_{2}, z_{3}\right) \mapsto\left(y_{2}, y_{3}, y_{1}, z_{2}, z_{3}, z_{1}\right) .
\end{aligned}
$$

The orbits of $y_{1}$ and of $\frac{y_{1}-z_{3}}{1-y_{1} z_{2}} \frac{y_{2}-z_{1}}{1-y_{2} z_{3}} \frac{y_{3}-z_{2}}{1-y_{3} z_{1}} \quad$ under $\quad G^{\prime}$ have order 12 and 32 , respectively. As in the previous subsection, we introduce "parametrization variables" $t_{1}, \ldots, t_{4}$, subject to the constraint $\prod_{j=1}^{4} t_{j}=1$, and form 6 arguments

$$
\left\{A_{i}\right\}_{i=1,2,3}=\left\{t_{i} t_{4}\right\}_{i}, \quad\left\{B_{i}\right\}_{i=1,2,3}=\left\{\frac{1-t_{j}^{-1}}{1-t_{i}} \frac{1-t_{k}^{-1}}{1-t_{4}} \mid\{i, j, k\}=\{1,2,3\}\right\} .
$$


The involutory automorphism induced by

$$
\left(t_{1}, t_{2}, t_{3}, t_{4}\right) \mapsto\left(\iota\left(t_{4}, t_{1}\right), \iota\left(t_{3}, t_{2}\right), \iota\left(t_{2}, t_{3}\right), \iota\left(t_{1}, t_{4}\right)\right)
$$

where $\iota(x, y)=\frac{1-x}{1-y^{-1}}$, acts on $\left(B_{1}, B_{2}, B_{3}, A_{1}, A_{2}, A_{3}\right)$ like the automorphism $g$ in (3.2) above while the automorphism induced by $\left(t_{1}, t_{2}, t_{3}\right) \mapsto\left(t_{2}, t_{3}, t_{1}\right)$ acts like $h$ in $(3.3)$.

If we put $y_{i}$ and $z_{i}$ equal to $A_{i}$ and $B_{i}$, respectively, in the above, then the $G^{\prime}$-orbit of the expression $\frac{y_{1}-z_{3}}{1-y_{1} z_{2}} \frac{y_{2}-z_{1}}{1-y_{2} z_{3}} \frac{y_{3}-z_{2}}{1-y_{3} z_{1}}$ contains only 16 different terms up to inversion of the arguments - obviously, the $G^{\prime}$-orbit of $y_{1}$ contains only 6 terms up to inversion - and the resulting $16+6$ arguments coincide precisely with the ones occurring in Goncharov's equation.

3.1.4. Other descriptions. A different way to use the above parametrization with $A_{i}$ and $B_{i}$ in order to exhibit the symmetries among the 22 terms is obtained by putting $\alpha_{i}=-\sqrt{A_{i}}, \beta_{i}=\sqrt{B_{i}}$. Then the four elements $\alpha_{1}^{\varepsilon_{1}} \alpha_{2}^{\varepsilon_{2}} \alpha_{3}^{\varepsilon_{3}}$ with $\varepsilon_{j} \in\{ \pm 1\}$ $(j=1,2,3)$ and $\prod \varepsilon_{j}=-1$, together with the twelve elements $\alpha_{i}^{\varepsilon_{i}} \beta_{i+1}^{\varepsilon_{i+1}} \beta_{i+2}^{\varepsilon_{i+2}}$

$(i \bmod 3)$ with $\varepsilon_{j} \in\{ \pm 1\}$ and $\prod \varepsilon_{j}=1$, form the 16 arguments in the second orbit above in $\S 3.1 .3$, while the $\alpha_{i}^{2}$ and $\beta_{i}^{2}$ give the 6 arguments in the first orbit.

Yet another description - in terms of equations instead of parametrizations - is given by the following 9 (redundant) equations where we put $q(x, y)=\frac{x-y}{1-x y}$ :

$$
\begin{aligned}
& B_{i}^{-1}=\frac{q\left(B_{i-1}, B_{i+1}\right)}{q\left(A_{i+1}, A_{i-1}\right)}, \\
& A_{i}^{-1}=q\left(A_{i+1}, \frac{1}{B_{i-1}}\right) q\left(A_{i-1}, \frac{1}{B_{i+1}}\right), \\
& \frac{A_{i+1}\left(1-A_{i}\right)^{2}}{A_{i}\left(1-A_{i+1}\right)^{2}}=\frac{B_{i+1}\left(1-B_{i}\right)^{2}}{B_{i}\left(1-B_{i+1}\right)^{2}}
\end{aligned}
$$

where $i=1,2,3$ and the indices are taken modulo 3. (Obviously, we can break the symmetry and reduce this to a system of 7 equations in only 4 variables by eliminating, say, $A_{1}$ and $B_{1}$. The system of equations is no complete intersection, though.)

3.2. Relating Goncharov's and Wojtkowiak's equations. In order to compare the equations resulting from Goncharov's and Wojtkowiak's approach, we propose to study an "intermediate" relation, with 34 terms, which allows an interpretation for both situations.

1. In Wojtkowiak's equation, the "intermediate" relation occurs when we consider the rational function

$$
\phi(x)=\frac{x-a}{x-c^{-1}} \cdot \frac{x-b}{x-a b c}=\operatorname{cr}(x, x b c, b, a b c),
$$

(for $x=1$ this is a $B_{i}$-type term above) and $(A, B, C)=(\infty, 0,1)$, and subtracting two different specializations for $x$ of the ensuing terms from Corollary 3.2 - each specialization providing 17 terms - we are left with 34 terms which form a functional equation in three variables. The generic 17 terms are given, in factored form, as 
the arguments of the following formal linear combination in $\mathbb{Z}[F], F=\mathbb{Q}(a, b, c, t)$ :

$$
\begin{gathered}
f(a, b, c, t)=\left[\frac{(1-c t) a}{a-t}\right]+\left[\frac{(1-c t) b}{b-t}\right]+\left[\frac{1-c t}{c(a-t)}\right]+\left[\frac{1-c t}{c(b-t)}\right] \\
+\left[\frac{a b c-t}{(a-t) b c}\right]+\left[\frac{a b c-t}{(b-t) a c}\right]+\left[\frac{a b c-t}{a-t}\right]+\left[\frac{a b c-t}{b-t}\right] \\
+\left[\frac{(a-t) b(a c-1)}{(b-t) a(b c-1)}\right]+\left[\frac{(t-a)(1-b c)}{(t-b)(1-a c)}\right]+\left[\frac{(t c-1) b(a c-1)}{(a b c-t)(b c-1)}\right]+\left[\frac{(t c-1) a(b c-1)}{(a b c-t)(a c-1)}\right] \\
-\left[\frac{(1-c t) a b c}{a b c-t}\right]-\left[\frac{(1-c t)}{(a b c-t) c}\right]-\left[\frac{t-a}{t-b}\right]-\left[\frac{b(a-t)}{a(b-t)}\right]-\left[\frac{(b-t)(a-t) c}{(a b c-t)(1-c t)}\right] .
\end{gathered}
$$

Definition 3.4. The 34-term relation is given by the difference

$$
f(a, b, c, t)-f(a, b, c, u) \in \mathbb{Z}[F], \quad F=\mathbb{Q}(a, b, c, t, u) .
$$

Remark 3.5. From Corollary 3.2 it results that $\mathcal{L}_{3}$ vanishes on 34 -term relations.

2. Let us recall that Goncharov [4] has defined a triple ratio for 6 points in the projective plane over a field $F$, with values in $\mathbb{Z}[F]$, in such a way that it respects a 7 -term relation: for any 7 distinct points $P_{1}, \ldots, P_{7}$ in $\mathbb{P}^{2}$ one has

$$
\sum_{i=1}^{7}(-1)^{i} \cdot \text { triple } \operatorname{ratio}\left(P_{1}, \ldots, \widehat{P}_{i}, \ldots, P_{7}\right)=0 .
$$

In the setting of configurations, the 34-term relation mentioned above encodes the well-definedness of the triple ratio associated to a configuration of six points $P_{i}$ $(i=1, \ldots, 6)$ in general position in the plane as follows.

Goncharov reduces such a configuration with the help of the 7-term relation above to more degenerate configurations by introducing the intersection point $Q$ of the line through the points $P_{1}, P_{2}$ with the line passing through $P_{3}$ and $P_{4}$. He uses the fact that he has already defined the triple ratio for the more degenerate configurations which result by leaving out any other of the 6 original points $P_{i}$. But there are different possibilities for choosing $Q$, depending on the ordering of the $P_{i}$ - e.g., one could switch the roles of $P_{2}$ and $P_{3}$ - and one verifies (this is not explicitly done in [4]) that the difference of two such possibilities, if non-zero, essentially results in two sets of 17 terms which correspond precisely to the 34 terms in question. (Here "essentially" alludes to the fact that we argue modulo two simple functional equations for the trilogarithm, the inversion relation $\mathcal{L}_{3}(x)=\mathcal{L}_{3}(1 / x)$ and the 3 -term relation $\mathcal{L}_{3}(x)+\mathcal{L}_{3}(1 /(1-x))+\mathcal{L}_{3}(1-1 / x)=\mathcal{L}_{3}(1)$.)

3.3. Relating the 34-term and 22-term equation. In this subsection, we will indicate a proof of the following fact:

Proposition 3.6. The 22-term relation and the 34-term relation are equivalent in the sense that each one, together with its specializations, implies the other.

If we take the difference of the specializations of $f(a, b, c, t)$ to $t=1$ and $t=0$, respectively, then a number of terms degenerate and we are left with the sum of a Kummer-Spence relation and a 22-term relation. (Again, we work up to inversion and 3-term relation, the latter being a specialization of both 22-term and 34-term equation.) Similarly, the difference of the specializations to $t=a$ and $t=0$ gives a version of the Kummer-Spence relation itself. Thus the 34 -term relation implies the 22-term relation. Conversely, the substitution

$$
\left(t_{1}, t_{2}, t_{3}\right) \mapsto\left(\operatorname{cr}\left(t, 0, c^{-1}, a\right), \operatorname{cr}\left(t, 0, b, c^{-1}\right), \operatorname{cr}(t, 0, a, a b c)\right)
$$


maps the terms of the 22-term relation above in the form given in $\S 3.1 .2$ to the 17 terms of $f(a, b, c, t)$ together with five terms (plus a constant term) which are independent of $t$. This shows immediately that the 22-term relation implies the 34-term relation.

Combining the proposition with the previous subsection, we conclude:

Proposition 3.7. Goncharov's 22-term relation is subsumed in Wojtkowiak's family of equations in Corollary 3.2.

3.4. A 21-term equation. Yet another related equation of possible interest is a sum of four 22-term relations which combines to a functional equation in 3 variables with less, viz. only 21, different non-constant arguments (up to inversion), but with coefficients in $\{ \pm 1, \pm 2\}$ : with $\gamma$ as in (3.1), consider

$$
\Gamma(x, y, z)=\gamma\left(\frac{1}{1-x}, \frac{1-x}{1-x y}, 1-z\right)+\gamma\left(1-\frac{1}{x}, \frac{1-x y}{y(1-x)}, \frac{1}{1-z^{-1}}\right)
$$

and note that its symmetrization in the first 2 arguments, which of course also constitutes a functional equation for the trilogarithm, has the following form-we introduce a "symmetrizing variable" $z_{2}=\left(x_{1} x_{2} z_{1}\right)^{-1}$ and put $j(t, u)=\frac{1-u^{-1}}{1-t}$ :

$$
\begin{aligned}
& \Gamma\left(x_{1}, x_{2}, z_{1}\right)+\Gamma\left(x_{2}, x_{1}, z_{1}\right)=-2\left[x_{1} x_{2}\right]-2[1] \\
& +2 \sum_{i=1}^{2}\left(\left[x_{i}\right]+\left[j\left(x_{i}, x_{3-i}\right)\right]+\left[z_{i}\right]+\left[j\left(z_{i}, z_{3-i}\right)\right]\right. \\
& \left.\quad-\left[x_{i} j\left(z_{1}, z_{2}\right)\right]-\left[j\left(x_{i}, x_{3-i}\right) j\left(z_{1}, z_{2}\right)\right]-\left[x_{i} z_{1}\right]-\left[j\left(x_{i}, x_{3-i}\right) z_{1}\right]\right) \\
& \quad+\sum_{i=1}^{2}\left(\left[x_{i} z_{1} j\left(x_{i}, x_{3-i}\right) j\left(z_{1}, z_{2}\right)\right]+\left[j\left(x_{i}, x_{3-i}\right) j\left(z_{1}, z_{2}\right) /\left(x_{i} z_{1}\right)\right]\right) .
\end{aligned}
$$

\section{4-LOGARITHM EQUATIONS}

It is in general a tedious job to verify even a single functional equation for the polylogarithms. Therefore it seems worthwhile finding a rather short way to verify a whole family of them. We propose to do this for a family of equations for the 4-logarithm, using essentially only one (and actually trivially checked) polynomial equation. One can proceed similarly (albeit in a somewhat more complicated fashion) with other families (cf. [3], Thm. 4.4 and Thm. 4.9), even up to $n=6$. Let us emphasize that not only had there been no example for $n \geq 6$ previously known (apart from the trivial distribution relations) but Wechsung [10] even proved that the type of equation (called "Kummer-type") which gave the only examples for $n=4,5$ known at the time is not "good enough" for $n \geq 6$. The following example, which is proved in $\S 6$, is not of Kummer-type.

Theorem 4.1. Let $F$ be a field of characteristic $0, \phi(x)=x^{n-1}(x-1)$ for some $n \in \mathbb{N}$. For some $t, u \in F$, let $\left\{x_{i}\right\}_{i=1}^{n}=\phi^{-1}(t)$ resp. $\left\{y_{i}\right\}_{i=1}^{n}=\phi^{-1}(u)$ be the sets of preimages in some finite extension field $F^{\prime}$ of $F$. Then the following element in $\mathbb{Z}\left[F^{\prime}\right]$ is annihilated by $\mathcal{L}_{4}$ :

$$
\begin{aligned}
n(n-2)[ & \left.\frac{\prod_{i} x_{i}}{\prod_{j} y_{j}}\right]-(n-1)^{2} \sum_{i, j=1}^{n}\left[\frac{1-x_{i}^{-1}}{1-y_{j}^{-1}}\right]+n^{2} \sum_{i, j=1}^{n}\left[\frac{1-x_{i}}{1-y_{j}}\right] \\
& -n^{2}(n-1)^{2} \sum_{i, j=1}^{n}\left[\frac{x_{i}}{y_{j}}\right]+n(n-1)^{2} \sum_{i=1}^{n}\left(\left[1-\frac{1}{x_{i}}\right]-\left[1-\frac{1}{y_{i}}\right]\right) .
\end{aligned}
$$




\section{A 7-LOGARITHM EQUATION}

In this section we give a 2 -variable functional equation for the 7 -logarithm. This functional equation consists of 274 terms, but by using an appropriately symmetrized notation we can write it in a more digestible form.

5.1. Let $F$ be the rational function field in two variables $\mathbb{Q}(t, u)$. Put

$$
\begin{aligned}
f_{1}(z)= & \frac{-z}{1-z+z^{2}}, \quad f_{2}(z)=\frac{z-1}{1-z+z^{2}}, \quad f_{3}(z)=\frac{z(1-z)}{1-z+z^{2}} \\
& \text { and } \quad f(z)=-f_{1}(z) f_{2}(z) f_{3}(z)=\frac{z^{2}(1-z)^{2}}{\left(1-z+z^{2}\right)^{3}} .
\end{aligned}
$$

Notice that the group $G \cong \mathcal{S}_{3}$ generated by $z \rightarrow 1 / z$ and $z \rightarrow 1-z$ permutes the $f_{i}$ and leaves $f$ invariant. For $a, b, c, d \in \mathbb{Z}$ define elements $\{a, b ; c, d\}_{0}(t, u)$ and $\{a, b ; c, d\}$ in $\mathbb{C}[\mathbb{C}]$ by

$$
\begin{array}{r}
\{a, b ; c, d\}_{0}(t, u)=\sum_{i, j=1}^{3}\left[\frac{f(t)^{a} f_{i}(t)^{b-a}}{f(u)^{c} f_{j}(u)^{d-c}}\right] \text { and } \\
\{a, b ; c, d\}=\{a, b ; c, d\}_{0}(t, u)+\{c, d ; a, b\}_{0}(t, u) \\
=\{a, b ; c, d\}_{0}(t, u)+\{a, b ; c, d\}_{0}(u, t),
\end{array}
$$

where the latter equation holds only up to inversions of the arguments (which, of course, does not affect the functional equation to be presented below). Finally, set

$$
\begin{aligned}
& \xi_{7}^{(3)}=-\frac{1}{18} \cdot \frac{609}{4}\{-1,-1 ;-1,-1\}-\frac{1}{3} \cdot 35\{-1,-1 ;-2,1\}+\frac{1}{3} \cdot \frac{105}{8}\{-1,-1 ; 3,-5\} \\
& -\frac{1}{3} \cdot 21\{-1,-1 ;-1,4\}-\frac{1}{3} \cdot 15\{-1,-1 ;-2,5\}+\frac{1}{3} \cdot 15\{-1,-1 ; 3,-4\}, \\
& \xi_{7}^{(2)}=+\frac{1}{2} \cdot 700\{1,0 ; 1,0\}+\frac{1}{2} \cdot \frac{175}{4}\{1,-3 ; 1,-3\}+\frac{1}{2} \cdot 28\{-2,3 ;-2,3\} \\
& -35\{1,-3 ;-2,3\}-140\{-2,3 ; 1,0\}+175\{1,0 ; 1,-3\} \text {, } \\
& \xi_{7}^{(1)}=+\frac{1}{2} \cdot 700\{1,-2 ;-1,2\}+3150\{0,1 ; 1,-1\}+\frac{1}{2} \cdot 1575\{-1,1 ; 1,-1\} \\
& -2100\{1,-2 ; 0,-1\}+\frac{1}{2} \cdot 6300\{0,1 ; 0,-1\}-1050\{-1,2 ;-1,1\} \\
& -\frac{1}{2} \cdot 700\{-1,2 ;-1,2\}-\frac{1}{2} \cdot 1575\{-1,1 ;-1,1\}-\frac{1}{2} \cdot 6300\{0,1 ; 0,1\} \\
& +1050\{-1,2 ; 1,-1\}+2100\{0,-1 ;-1,2\}-3150\{1,-1 ; 0,-1\} \text {. }
\end{aligned}
$$

Theorem 5.1. The element $\xi_{7}=\xi_{7}^{(1)}+\xi_{7}^{(2)}+\xi_{7}^{(3)} \in \mathbb{Q}[F]$ is a functional equation for $\mathcal{L}_{7}$, i.e.,

$$
\mathcal{L}_{7}\left(\xi_{7}(t, u)\right)=0
$$

for any $t, u \in \mathbb{C}$ where $f_{j}(t), f_{j}(u) \notin\{0, \infty\}, j=1,2,3$.

Proof. Functional equations for polylogarithms can be characterized by an algebraic criterion (cf. [14], Proposition 1), so the proof can be reduced to checking this criterion for $\xi_{7}$ which was done with a computer program. The statement applies, of course, to any functional equation; the difficulty lies in finding functional equations, not in checking their validity.

5.2. Remark. In $\xi_{7}$ the denominator of the first factor of a coefficient (which we suppress if it is 1) gives the multiplicity of an argument in the sum $\{a, b ; c, d\}$ (where we identify $[z]$ and $[1 / z]$ ).

We associate a weight to each $\{a, b ; c, d\}$ by $\operatorname{wt}(\{a, b ; c, d\})=\operatorname{wt}(a, b)$ in the following way: setting $\operatorname{wt}(a, b)=\frac{1}{2}(|a|+|a+b|+|2 a+b|)$ we find that in all terms $\{a, b ; c, d\}$ of $\xi_{7}$ we have $\operatorname{wt}(a, b)=\operatorname{wt}(c, d)$. 
This provides each $\xi_{7}^{(i)}(i=1,2,3)$ with weight $i$. There is a weight-preserving operation on these blocks which also includes the coefficients. We explain this operation in more detail in the following subsection.

5.3. More symmetric form of $\xi_{7}$. Let $\left(\mathbb{Z}^{3}\right)_{0}=\left\{(a, b, c) \in \mathbb{Z}^{3} \mid a+b+c=0\right\}$ and $\left(\mathbb{Z}^{3}\right)_{1}=\left\{(a, a, b) \in \mathbb{Z}^{3}\right\}$. We consider the map

$$
\begin{aligned}
\Theta: \mathbb{Z}^{3} & \rightarrow \mathbb{Z}^{3}, \\
(a, b, c) & \mapsto(a,-b-c, b-a)
\end{aligned}
$$

which sends $\left(\mathbb{Z}^{3}\right)_{0}$ to $\left(\mathbb{Z}^{3}\right)_{1}$, and a second one (the $f_{i}$ are taken as in $(5.1)$ )

$$
\begin{aligned}
\Phi: \mathbb{Z}^{3} & \rightarrow\left\{\phi: \mathbb{P}_{\mathbb{C}}^{1} \rightarrow \mathbb{P}_{\mathbb{C}}^{1} \mid \phi \text { rational }\right\}, \\
\alpha=\left(\alpha_{1}, \alpha_{2}, \alpha_{3}\right) & \mapsto\left(\phi_{\alpha}: z \mapsto\left(-f_{1}(z)\right)^{\alpha_{1}} f_{2}(z)^{\alpha_{2}} f_{3}(z)^{\alpha_{3}}\right) .
\end{aligned}
$$

The symmetric group $\mathcal{S}_{3}$ operates on $\left(\mathbb{Z}^{3}\right)_{0}$ and $\mathbb{Z}^{3}$ via permutation.

For each $k \in \mathbb{Z}$ we have the element $(k,-1,1-k) \in\left(\mathbb{Z}^{3}\right)_{0}$.

We set

$$
A_{k}=\Theta\left(\mathcal{S}_{3} \cdot(k,-1,1-k)\right), \quad k=1,2,3
$$

Then we have

$$
\begin{aligned}
& A_{1}=\{ \pm(1,1,-2), \pm(-1,-1,1), \pm(0,0,1)\} \\
& A_{2}=\{(2,2,-3),(-1,-1,3),(-1,-1,0)\} \\
& A_{3}=\{(-1,-1,-1),(-1,-1,4),(-2,-2,5),(-2,-2,1),(3,3,-4),(3,3,-5)\} .
\end{aligned}
$$

We will denote by $\delta$ the $\mathcal{S}_{3}$-invariant element $(-1,-1,-1)$ of $A_{3}$ which will play a special role. Finally we define for $\alpha=\left(\alpha_{1}, \alpha_{2}, \alpha_{3}\right) \neq 0$ a "weight"

$$
\omega(\alpha)=\frac{1}{\alpha_{1}-\alpha_{3}} .
$$

Then Theorem 5.1 can be restated more concisely by saying that

$$
60 \xi_{7}=\sum_{i=1}^{3} \xi^{(i)}
$$

with

$$
\begin{aligned}
& \xi^{(1)}=-\frac{29}{20}\left[\frac{\phi_{\delta}(t)}{\phi_{\delta}(u)}\right]+\sum_{\alpha \in A_{3} \backslash\{\delta\}} \omega(\alpha) \sum_{\sigma \in \mathcal{S}_{3} / \mathcal{S}_{2}}\left(\left[\frac{\phi_{\sigma \alpha}(t)}{\phi_{\delta}(u)}\right]+\left[\frac{\phi_{\delta}(t)}{\phi_{\sigma \alpha}(u)}\right]\right), \\
& \xi^{(2)}=\frac{20}{3} \sum_{\alpha, \beta \in A_{2}} \omega(\alpha) \omega(\beta) \sum_{\sigma, \tau \in \mathcal{S}_{3} / \mathcal{S}_{2}}\left[\frac{\phi_{\sigma \alpha}(t)}{\phi_{\tau \beta}(u)}\right], \\
& \xi^{(3)}=-30 \sum_{\alpha, \beta \in A_{1}} \omega(\alpha) \omega(\beta) \sum_{\sigma, \tau \in \mathcal{S}_{3} / \mathcal{S}_{2}}\left[\frac{\phi_{\sigma \alpha}(t)}{\phi_{\tau \beta}(u)}\right] .
\end{aligned}
$$

Altogether we get $1+30+81+162=274$ different arguments (up to inverses) since in the last sum (over $\left.A_{1}\right)$ the arguments associated to $(\alpha, \beta)$ and $(-\alpha,-\beta)$ are inverse to each other. 


\section{A Proof of Theorem 4.1}

Proof. Using Zagier's criterion (cf. [14], Proposition 1) alluded to earlier, we need only verify that the above combination lies in the kernel of the map

$$
\begin{aligned}
\beta_{4}: \mathbb{Z}\left[F^{\prime}\right] & \longrightarrow \operatorname{Sym}^{2}{F^{\prime}}^{\times} \otimes \bigwedge^{2}{F^{\prime}}^{\times} \\
{[x] } & \longmapsto x^{\odot 2} \otimes(x \wedge(1-x)) .
\end{aligned}
$$

Here the $\bigwedge^{2}$ denotes the second exterior product (i.e., two-fold tensors subject to the relations $x \wedge x=0)$. Note that $x y \wedge z=x \wedge z+y \wedge z$.

Our strategy is as follows: a few preliminary considerations (steps 0-2) allow to rewrite the $\beta_{4}$-images in a more convenient way, so that the theorem is essentially reduced to Claim 6.1 (in step 3).

Part I: Reformulations. 0 . Note that $t-\phi(z)$ is a polynomial in (the variable) $z$ with roots equal to $\left\{x_{i}\right\}_{i}$, therefore

$$
t-\phi(z)=\phi\left(x_{i}\right)-\phi(z)=\lambda \prod_{i}\left(x_{i}-z\right)
$$

for some constant $\lambda$. Thus, for any fixed $l$ and $m$,

$$
t-u=\phi\left(x_{l}\right)-\phi\left(y_{m}\right)=\lambda \prod_{i}\left(x_{i}-y_{m}\right)=\mu \prod_{j}\left(x_{l}-y_{j}\right)
$$

for some constants $\lambda$ and $\mu$ (which actually turn out to be equal to \pm 1 and therefore can be neglected in the following).

1. As a first preliminary step, we express $\alpha$ and $1-\alpha$ in terms of the factors $x_{i}$ and $y_{j}$ for each of the arguments $\alpha$ in (4.1). In order to save indices, we put $x=x_{l}$ and $y=y_{m}$ for some fixed $l, m \in\{1, \ldots, n\}$. We obtain

$$
\frac{1-x}{1-y}=\frac{y^{n-1} X}{x^{n-1} Y} \quad \text { and } \quad \frac{1-x^{-1}}{1-y^{-1}}=\frac{y^{n} X}{x^{n} Y},
$$

where we have set $X=\prod_{i} x_{i}, Y=\prod_{j} y_{j}$. A further decomposition we need is

$$
1-\frac{1-x}{1-y}=\frac{y^{n-1}}{Y}(x-y) \quad \text { and } \quad 1-\frac{1-x^{-1}}{1-y^{-1}}=\frac{y^{n-1}}{x Y}(x-y) .
$$

With these preparations, we can write the corresponding images under $\beta_{4}$ as

$$
\beta_{4}\left(\left[\frac{1-x}{1-y}\right]\right)=\left(\frac{y^{n-1} X}{x^{n-1} Y}\right)^{\otimes 2} \otimes \frac{y^{n-1} X}{x^{n-1} Y} \wedge \frac{y^{n-1}}{Y}(x-y)
$$

and

$$
\beta_{4}\left(\left[\frac{1-x^{-1}}{1-y^{-1}}\right]\right)=\left(\frac{y^{n} X}{x^{n} Y}\right)^{\otimes 2} \otimes \frac{y^{n} X}{x^{n} Y} \wedge \frac{y^{n-1}}{x Y}(x-y) .
$$

These two expressions decompose "naturally" into two parts, one of which contains the factor $(x-y)$ in the last tensor factor. E.g., the part in $\bigwedge^{2} F^{\prime \times}$ in the first expression (6.1) factors as

$$
\frac{y^{n-1} X}{x^{n-1} Y} \wedge \frac{y^{n-1}}{Y}(x-y)=\frac{X}{x^{n-1}} \wedge \frac{y^{n-1}}{Y}+\frac{y^{n-1} X}{x^{n-1} Y} \wedge(x-y)
$$

where the first summand on the right has been reduced using the defining property of $\wedge$.

2. As a second preliminary step, we "switch" to an additive notation-formally, we put $\xi_{i}=" \log x_{i} ", \eta_{j}=" \log y_{j} "$, and introduce $\zeta_{l m}=" \log \left(x_{l}-y_{m}\right) "$ as well as the shorthands $\xi=\sum_{i} \xi_{i}$ and $\eta=\sum_{j} \eta_{j}$ (note also that the $\wedge$ now satisfies 
$(a+b) \wedge c=a \wedge c+b \wedge c)$; then the term (6.1) resp. (6.2) is written, using the decomposition (6.3), as

$$
\begin{aligned}
& \left((n-1)\left(\eta_{m}-\xi_{l}\right)+\xi-\eta\right)^{3} \wedge \zeta_{l m} \\
& \quad+\left((n-1)\left(\eta_{m}-\xi_{l}\right)+\xi-\eta\right)^{2} \cdot\left(\xi-(n-1) \xi_{l}\right) \wedge\left(-\eta+(n-1) \eta_{m}\right)
\end{aligned}
$$

resp.

$$
\begin{aligned}
& \left(n\left(\eta_{m}-\xi_{l}\right)+\xi-\eta\right)^{3} \wedge \zeta_{l m} \\
& +\left(n\left(\eta_{m}-\xi_{l}\right)+\xi-\eta\right)^{2} \cdot\left(\xi-(n-1) \xi_{l}+\eta_{m}\right) \wedge\left(-\eta+(n-1) \eta_{m}-\xi_{l}\right) .
\end{aligned}
$$

Introducing the shorthands $S=\xi-\eta$ and $s_{l m}=\xi_{l}-\eta_{m}$, we rewrite the latter two equations as

(6.6) $\left(S-(n-1) s_{l m}\right)^{3} \wedge \zeta_{l m}+\left(S-(n-1) s_{l m}\right)^{2} \cdot\left(\xi-(n-1) \xi_{l}\right) \wedge\left(-\eta+(n-1) \eta_{m}\right)$

resp.

(6.7) $\left(S-n s_{l m}\right)^{3} \wedge \zeta_{l m}+\left(S-n s_{l m}\right)^{2} \cdot\left(\xi-(n-1) \xi_{l}+\eta_{m}\right) \wedge\left(-\eta+(n-1) \eta_{m}-\xi_{l}\right)$.

Part II: Calculations. 3. So far, we have only reformulated the objects under consideration. Now we can proceed to the two actual calculations involved, the first of which follows easily from the readily verified identity

$$
\left(\xi-(n-1) \xi_{l}\right) \wedge\left(\eta-(n-1) \eta_{m}\right)=\sum_{i, j}(2-n)^{\delta_{i l}+\delta_{j m}} \xi_{i} \wedge \eta_{j} .
$$

Here $\delta_{i j}(=1$ if $i=j$, and $=0$ otherwise) denotes the usual "Kronecker- $\delta$ ".

Using relation (6.8) together with the presentations (6.6) and (6.7), the $\beta_{4}$-image of the first two terms in (4.1) becomes

$$
\beta_{4}\left(n^{2}\left[\frac{1-x_{l}}{1-y_{m}}\right]-(n-1)^{2}\left[\frac{1-x_{l}^{-1}}{1-y_{m}^{-1}}\right]\right)=T_{1}^{l m}+T_{2}^{l m}+T_{3}^{l m}+T_{4}^{l m}
$$

where

$$
\begin{aligned}
& T_{1}^{l m}=\left((2 n-1) S^{3}-3 n(n-1) S^{2} s_{l m}+n^{2}(n-1)^{2} s_{l m}^{3}\right) \wedge \zeta_{l m}, \\
& T_{2}^{l m}=-\left(n^{2}\left(S-(n-1) s_{l m}\right)^{2}-(n-1)^{2}\left(S-n s_{l m}\right)^{2}\right)\left(\sum_{i, j}(2-n)^{\delta_{i l}+\delta_{j m}} \xi_{i} \wedge \eta_{j}\right), \\
& T_{3}^{l m}=(n-1)^{2}\left(S-n s_{l m}\right)^{2} \cdot\left(\eta_{m} \wedge \xi_{l}\right), \\
& T_{4}^{l m}=(n-1)^{2}\left(S-n s_{l m}\right)^{2} \cdot\left(\xi \wedge \xi_{l}+\eta_{m} \wedge \eta\right) .
\end{aligned}
$$

We will sum the latter expression over all $l$ and $m$ (unless explicitly indicated otherwise, all sums in the following run from 1 to $n$ ).

Claim 6.1. With notations as above, we have

$$
\begin{aligned}
\sum_{l, m} \beta_{4}\left(n^{2}\left[\frac{1-x_{l}}{1-y_{m}}\right]\right. & \left.-(n-1)^{2}\left[\frac{1-x_{l}^{-1}}{1-y_{m}^{-1}}\right]\right) \\
= & n(n-2)\left(-S^{3} \wedge\left(\frac{1}{n} \sum_{l, m} \zeta_{l m}\right)+S^{2} \sum_{l, m} \xi_{l} \wedge \eta_{m}\right) \\
& +n^{2}(n-1)^{2}\left(\sum_{l, m} s_{l m}^{3} \wedge \zeta_{l m}-\sum_{l, m} s_{l m}^{2}\left(\xi_{l} \wedge \eta_{m}\right)\right) \\
& +\sum_{i} f_{1}\left(\xi_{i}\right)+\sum_{j} f_{2}\left(\eta_{j}\right)
\end{aligned}
$$

for some functions $f_{k}(k=1,2)$ depending on only one of the variables $t$ and $u$. 
Proof. We will need the following immediately verified identities:

$$
\begin{gathered}
Z:=\frac{1}{n} \sum_{i, j} \zeta_{i j}=\sum_{i} \zeta_{i m}=\sum_{j} \zeta_{l j}, \quad \forall l, \forall m \\
S=\sum_{i} \xi_{i}-\sum_{j} \eta_{j}=\frac{1}{n} \sum_{i, j} s_{i j} \\
\sum_{m}\left(\xi-n \xi_{m}\right)=\sum_{l}\left(\eta-n \eta_{l}\right)=0
\end{gathered}
$$

and the "distribution properties" (for fixed $l, m$ ):

$$
\sum_{i, j}(2-n)^{\delta_{i l}+\delta_{j m}}=\sum_{i}(2-n)^{\delta_{i l}}=1
$$

as well as some simple consequence of the above

$$
\sum_{l, m} s_{l m}(2-n)^{\delta_{i l}+\delta_{j m}}=S-(n-1) s_{i j} .
$$

[Proof: write $s_{l m}=\xi_{l}-\eta_{m}$ and use successively the equations in (6.13).]

We will first treat the sum $\sum_{l, m} T_{4}^{l m}$ which is easily reduced to the form $\sum_{i} f_{1}\left(\xi_{i}\right)+$ $\sum_{j} f_{2}\left(\eta_{j}\right)$ since each of its terms which is dependent on both $\xi$ 's and $\eta$ 's is cancelled in the sum by virtue of (6.12).

Thus it will suffice to identify the sum $\sum_{i=1}^{3} \sum_{l, m} T_{i}^{l m}$ with the first two lines on the right hand side of the claim. The first two terms of

$$
\begin{aligned}
\sum_{l, m} T_{1}^{l m}=(2 n-1) S^{3} \wedge \sum_{l, m} \zeta_{l m}-3 n(n-1) S^{2} & \sum_{l, m} s_{l m} \wedge \zeta_{l m} \\
& +n^{2}(n-1)^{2} \sum_{l, m} s_{l m}^{3} \wedge \zeta_{l m}
\end{aligned}
$$

combine to

$$
-n(n-2) S^{3} \wedge Z
$$

(note that $\sum_{l, m} s_{l m} \wedge \zeta_{l m}=S \wedge Z$ by using (6.10) and (6.11)), while the third term on the right of (6.15) is already of the desired form.

The term

$$
\sum_{l, m} T_{2}^{l m}=-\sum_{l, m}\left((2 n-1) S^{2}-2 n(n-1) S s_{l m}\right) \sum_{i, j}(2-n)^{\delta_{i l}+\delta_{j m}} \xi_{i} \wedge \eta_{j}
$$

decomposes into two simpler sums, the first of which is given as

$$
-(2 n-1) S^{2} \sum_{i, j} \sum_{l, m}(2-n)^{\delta_{i l}+\delta_{j m}} \xi_{i} \wedge \eta_{j}=-(2 n-1) S^{2} \sum_{i, j} \xi_{i} \wedge \eta_{j},
$$

where we have used (6.13), while the second one equals

$$
\begin{aligned}
2 n(n-1) S \sum_{l, m} s_{l m} \sum_{i, j}(2-n)^{\delta_{i l}+\delta_{j m}} \xi_{i} \wedge \eta_{j} & = \\
& 2 n(n-1) S^{2} \sum_{i, j} \xi_{i} \wedge \eta_{j}-2 n(n-1)^{2} S \sum_{i, j} s_{i j}\left(\xi_{i} \wedge \eta_{j}\right)
\end{aligned}
$$

by virtue of (6.14). Finally, expanding $\sum_{l, m} T_{3}^{l m}$ as a "polynomial" in $S$ gives us three further sums, one being quadratic, one linear and one constant in $S$; the quadratic one combines with (6.16) and with the first term on the right in (6.17) to $n(n-2) S^{2} \sum_{i, j} \xi_{i} \wedge \eta_{j}$, the linear one cancels with the second term in (6.17), and 
the constant one is given by $-n^{2}(n-1)^{2} \sum_{l, m} s_{l m}^{2}\left(\xi_{l} \wedge \eta_{m}\right)$. This proves Claim 6.1 .

4. We finish by observing that the first two terms on the right hand side of Claim 6.1 correspond precisely to the images under $\beta_{4}$ of $-n(n-2)\left[\prod x_{i} / \prod y_{j}\right]$ and $n^{2}(n-1)^{2} \sum_{i, j}\left[x_{i} / y_{j}\right]$, respectively, while $\sum_{l, m} T_{4}^{l m}$, which corresponds to the last two terms of the claim, can be recognized readily - after more careful investigation of the terms "purely in $\xi_{i}$ or $\eta_{j}$ " - as the image under $\beta_{4}$ of $-n(n-1)^{2}\left(\sum_{i}[1-\right.$ $\left.\left.x_{i}^{-1}\right]-\sum_{j}\left[1-y_{j}^{-1}\right]\right)$.

Acknowledgements: We would like to heartily thank Don Zagier for not only suggesting the original problem, but even more for his steady support, encourage- and enlightenment, and constant flow of ideas surrounding this work. It was made possible through the generosity and hospitality of the Max-Planck-Institut für Mathematik in Bonn with its unsurpassed working conditions.

\section{REFERENCES}

[1] Bloch, S. Higher regulators, algebraic K-theory, and zeta functions of elliptic curves, CRM Monograph Series, 11. American Mathematical Society, Providence, RI, 2000.

[2] Gangl, H., Funktionalgleichungen von Polylogarithmen, Thesis Bonn, Bonner Math. Schriften 278 (1995).

[3] Gangl, H., Families of functional equations for polylogarithms, Contemp. Math. 199, 83-105 (1996).

[4] Goncharov, A.B., Geometry of configurations, polylogarithms and motivic cohomology, Adv. Math. 114 (1995), 197-318.

[5] Kummer, E.E. Über die Transcendenten, welche aus wiederholten Integrationen rationaler Funktionen entstehen, JRAM (Crelle) 21 (1840), 74-90.

[6] Lewin, L., Polylogarithms and associated functions, North-Holland, New York, 1981.

[7] Lewin, L. (ed.), Structural properties of polylogarithms, Math. Surveys Monogr. 37, AMS, Providence, 1991.

[8] Lewin, L., The order-independence of the polylogarithmic ladder structure-implications for a new category of functional equations, Aequationes Math. 30 (1986), no. 1, 1-20.

[9] Lewin, L., Rost, E., Polylogarithmic functional equations: a new category of results developed with the help of computer algebra (MACSYMA), Aequationes Math. 31 (1986), no. 2-3, 223242.

[10] Wechsung, G., Kummer-type functional equations of polylogarithms, Chapter 9 in [7], 185203.

[11] Wechsung, G., Lineare Funktionalgleichungen von Polylogarithmen, Wiss. Z. FriedrichSchiller-Univ. Jena, Math.-Naturw. Reihe 14 (1965), 401-408.

[12] Wojtkowiak, Z., The basic structure of functional equations for polylogarithms, Chapter 10 in [7], 205-231.

[13] Wojtkowiak, Z., Functional equations of iterated integrals with regular singularities, Nagoya Math. J. 142 (1996), 145-159.

[14] Zagier, D., Polylogarithms, Dedekind zeta functions and the algebraic $K$-theory of fields, in Arithmetic Algebraic Geometry (G. v.d.Geer, F. Oort, J. Steenbrink, eds.), Prog. in Math. 89, Birkhäuser, Boston (1991), 391-430.

[15] Zagier, D., Special values and functional equations of polylogarithms, Appendix A in [7], $377-400$.

[16] Zagier, D., The remarkable dilogarithm, J. Math. Phys. Sci. 22 (1988), no. 1, 131-145.

MPI Bonn, Vivatsgasse 7, D-53111 Bonn, Germany

E-mail address: herbert@mpim-bonn.mpg.de 